\title{
Cartographier Fort-de-France à partir de sources historiques et littéraires : pour une géohistoire postcoloniale
}

Mapping Fort-de-France from Historical and Literary Sources: Toward a

Postcolonial Geohistory

\section{Juliette Morel}

\section{OpenEdition \\ Journals}

Édition électronique

URL : https://journals.openedition.org/revuehn/1503

DOI : 10.4000/revuehn.1503

ISSN : 2736-2337

Éditeur

Humanistica

\section{Référence électronique}

Juliette Morel, «Cartographier Fort-de-France à partir de sources historiques et littéraires : pour une géohistoire postcoloniale », Humanités numériques [En ligne], 3| 2021, mis en ligne le 01 mai 2021 consulté le 16 juillet 2021. URL : http://journals.openedition.org/revuehn/1503 ; DOI : https://doi.org/ 10.4000/revuehn. 1503

\section{(c) (1)}

Les contenus de la revue Humanités numériques sont mis à disposition selon les termes de la Licence Creative Commons Attribution 4.0 International. 


\section{humanités numériques}

3 | 2021

Humanités numériques spatialisées

INTÉGRATION ET USAGES DES DONNÉES HISTORIQUES ET PATRIMONIALES

\section{Cartographier Fort-de-France à partir de sources historiques et littéraires : pour une géohistoire postcoloniale}

Mapping Fort-de-France from Historical and Literary Sources: Toward a Postcolonial Geohistory

\section{Juliette Morel}

\section{Résumés}

À l'heure où se multiplient les systèmes d'information de reconstruction géographique et historique ayant pour fondement la numérisation des archives cartographiques et scientifiques, il est nécessaire de développer des méthodes spécifiques concernant les espaces anciennement colonisés, car ils posent des questions de sources et de modélisation particulières, marquées notamment par la nécessité d'adopter en géomatique une perspective critique, délicate, voire paradoxale. Les expérimentations interdisciplinaires réalisées dans le champ encore naissant des humanités numériques spatialisées offrent des pistes pour répondre à l'apparent paradoxe de la géomatique en contexte postcolonial. Cet article propose de démontrer la pertinence de cette hypothèse méthodologique en développant l'exemple de la ville de Fort-de-France en Martinique. L'objectif est de montrer que l'intégration d'informations géohistoriques alternatives, comme celles issues des romans, enrichit la modélisation géographique et historique de la ville. Notre intention est également d'ouvrir un chantier de recherche critique fondé sur l'enrichissement des informations géographiques par les humanités numériques, d'en montrer la pertinence et la légitimité et de soulever les questions qui le traversent. 
At a time when information systems for geographic and historical reconstruction based on the digitization of cartographic and scientific archives proliferate, it is necessary to develop methods for the study of formerly colonized spaces. These spaces raise specific problems in the treatment of sources and the modelling process : they require a geomatic perspective which has to be critical, delicate, and even paradoxical. Interdisciplinary experiments carried out in the still nascent field of spatialized digital humanities offer leads for responding to the apparent paradox of geomatics in postcolonial and decolonial contexts. This article aims at demonstrating the relevance of such methodological hypotheses by developing the example of the city of Fort-de-France in Martinique. The purpose of this paper is to illustrate that the integration of alternative geohistorical information, such as that derived from novels, enriches the geographic and historical modelling of the city. Our intention is to open a critical research project based on the enrichment of geographic information by the digital humanities, to show its relevance and legitimacy and to raise the questions that appear in such work.

\section{Entrées d'index}

MOTS-CLÉS : humanités numériques spatialisées, géographie, histoire, littérature, corpus d'auteur, roman, système d'information géographique

KEYWORDS: spatial digital humanities, geography, history, literature, authorial corpus, novel, geographic information system 


\section{Introduction}

La modélisation d'un espace dans la durée est pragmatiquement contrainte par les observations disponibles et les sources accessibles concernant cet espace (Mathian et Sanders 2014, 15). Il faut donc avoir conscience qu'une modélisation critique est dépendante du contexte de leur production et veiller à évaluer chaque information qu'elle peut en tirer. Or en contexte colonial et postcolonial, les conditions de production du savoir sur l'espace, en particulier des cartes, sont marquées par des enjeux de domination et de pouvoir, encore plus qu'ailleurs (Harley 2001 ; Blais 2014). Aujourd'hui se multiplient les projets transdisciplinaires de modélisation spatiotemporelle d'espaces délimités, souvent des villes, à partir de la digitalisation des archives cartographiques et des savoirs scientifiques ${ }^{1}$ - que nous désignerons sous l'expression générique de "systèmes d'information géographique et historique " (SIG-H). Ces projets ont pour objectif de rassembler, croiser, valoriser et rendre disponibles des ressources documentaires précieuses et les données qu'il est possible d'en tirer, et ainsi d'offrir des outils pour produire de nouvelles connaissances historiques et géographiques sur ces villes. Développer de tels outils géomatiques dans le cadre d'espaces anciennement colonisés nécessite des méthodes spécifiques, car il faut redoubler de vigilance critique, premièrement concernant les sources et les données elles-mêmes, et deuxièmement concernant leur structuration et traitement en vue de leur intégration dans de nouveaux systèmes de connaissance numérique. Nous voulons ici rendre compte d'une expérimentation s'intégrant dans ce cadre et visant à l'élaboration critique de jeux de données géohistoriques numériques sur une ville marquée par l'histoire coloniale. Cette expérimentation est fondée sur l'idée que l'ouverture du champ des sources de l'information géographique et historique numérique est possible, et nécessaire, pour que les systèmes d'information rendent compte de manière plus complexe et plus complète de l'histoire de ces villes, mais seulement au prix d'une méthodologie sans cesse réflexive. Autrement dit, la proposition est d'intégrer à part entière dans les SIG-H, en complément et en comparaison d'informations géographiques institutionnelles, des informations issues des représentations alternatives de l'espace : discours, récits, représentations artistiques ou subjectives. L'objectif de cet article est de donner un exemple très précis d'une telle démarche, afin d'en montrer la nécessité en même temps que la difficulté, d'en évaluer les bénéfices pratiques et d'identifier les impératifs méthodologiques qu'elle impose.

Pour ce faire, sera développé l'exemple précis de l'intégration des sources littéraires dans la cartographie historique de la ville de Fort-deFrance en Martinique. La modélisation historique de Fort-de-France apparaît comme un laboratoire idéal pour explorer des questions de géomatique critique du fait de l'histoire particulière de cette ville, de ses conditions de croissance au $\mathrm{xx}^{\mathrm{e}}$ siècle et de la place centrale qu'elle tient dans la culture et la littérature créole. En 2017, la commune s'étend sur $44 \mathrm{~km}^{2}$, est peuplée d'un peu plus de 80 ooo habitants et polarise une aire urbaine de 120 ooo habitants (figure 1). Ce n'est qu'à partir du début $\mathrm{du} \mathrm{xx}^{\mathrm{e}}$ siècle, après la destruction de la ville de Saint-Pierre par l'érup- 
tion volcanique de la montagne Pelée en 1902, que Fort-de-France s'impose comme la capitale politique ${ }^{2}$, économique et culturelle de la Martinique.

FIGURE 1. RÉSEAU VIAIRE ET PRINCIPAUX QUARTIERS DE LA VILLE DE FORT-DE-FRANCE AUJOURD'HUI

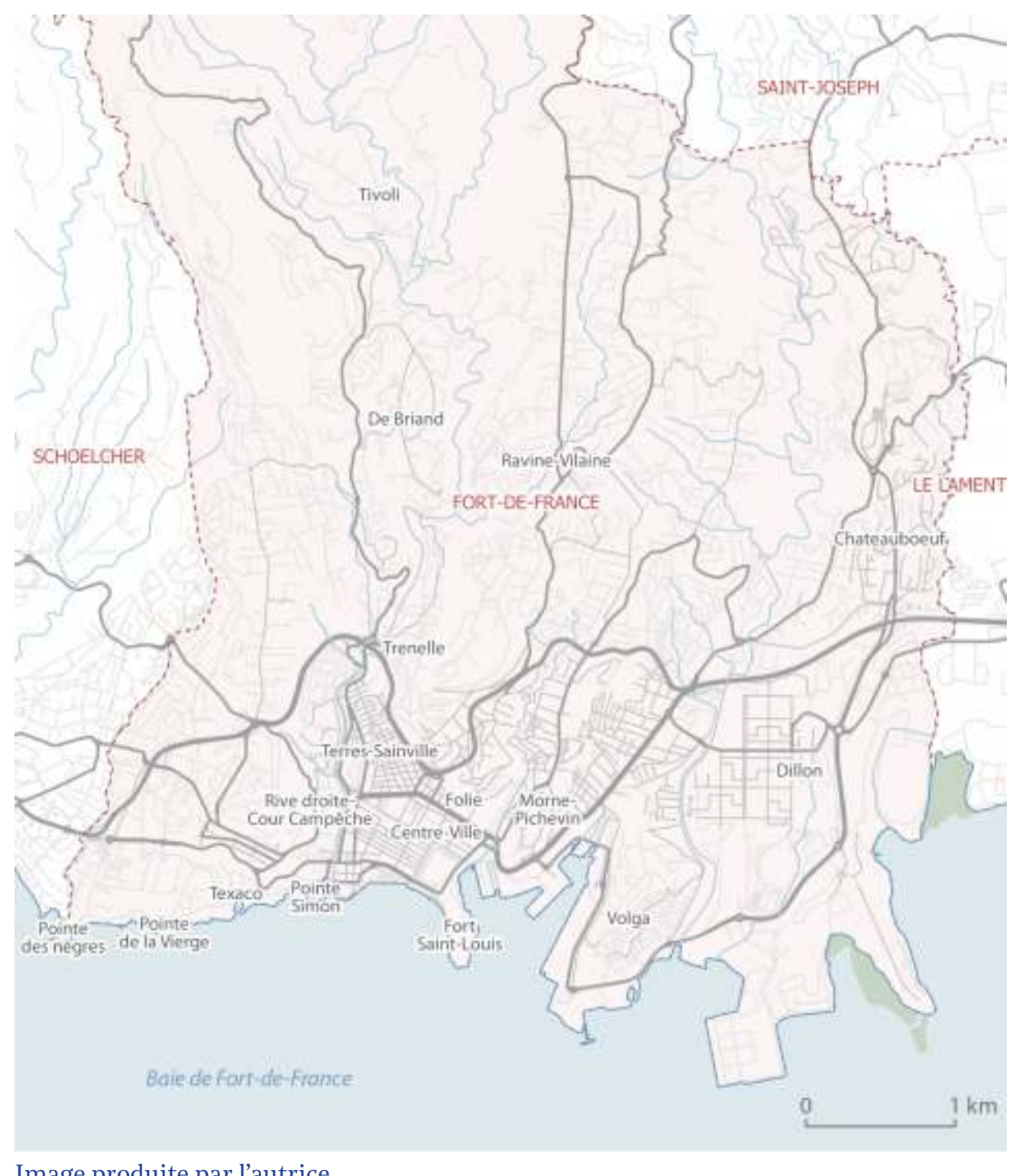

Image produite par l'autrice

\section{Exemple de la cartographie historique de Fort-de-France}

Parmi les sources privilégiées en histoire urbaine, les cartes tiennent une place centrale (Arnaud 2008). L'étude des sources cartographiques disponibles sur la ville de Fort-de-France fait néanmoins apparaître la nécessité d'ouvrir le champ des sources, tant parce que celles-ci sont insuffisantes, que parce que les sources alternatives, comme les représentations littéraires, sont extrêmement fertiles. 


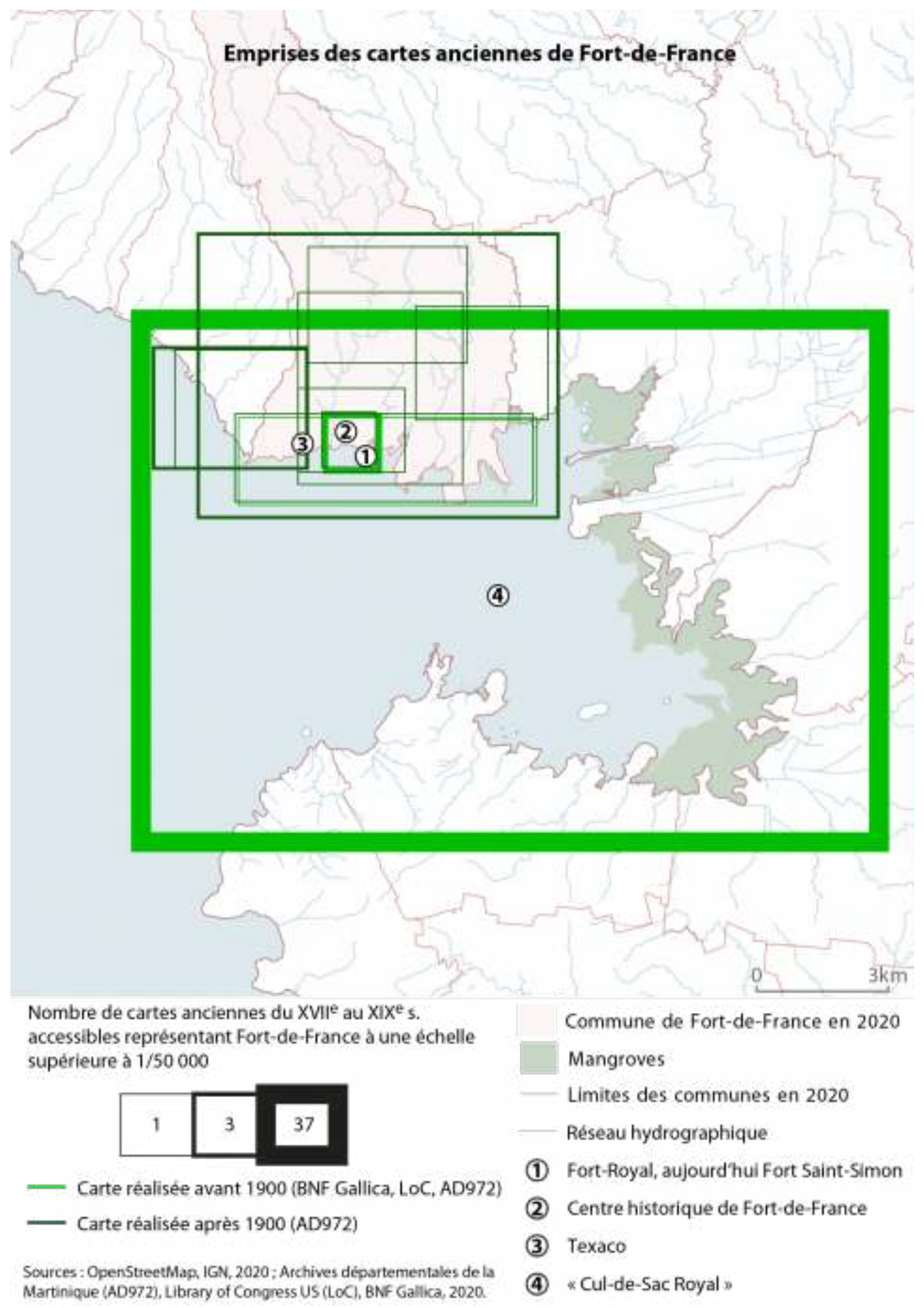

Image produite par l'autrice

L'étude de l'emprise des cartes anciennes représentant Fort-de-France est révélatrice du statut de la ville à différentes époques (figure 2). Avant la destruction de Saint-Pierre, la ville de Fort-de-France en tant que telle n'est pas au centre des cartes : la plupart des cartes du XviII ${ }^{\mathrm{e}}$ siècle sont centrées sur le "Cul-de-sac Royal » (la baie de Fort-de-France ${ }^{3}$ ), qui constitue à l'époque un espace stratégique pour accueillir les navires notamment militaires. La ville est représentée de manière schématique sur ces cartes (grisé, structure de la voirie, dessin schématique de maisons en perspective cavalière ou poncifs peu précis), voire n'apparaît pas du tout. Au contraire, le dessin des côtes et la profondeur de la baie sont précisément renseignés. Les autres cartes de la période se focalisent sur le centre historique de Fort-de-France, construit dans les années 1670 à l'ouest d'un fort militaire (anciennement Fort-Royal, nommé plus tard 
fort Saint-Louis). Au XIX ${ }^{\mathrm{e}}$ siècle, les cartes où apparaît Fort-de-France et qui sont conservées dans les fonds que nous avons pu consulter sont moins nombreuses ( 2 cartes contre 43 pour la période précédente). Bien que possiblement liée à des logiques de conservation, cette répartition dans le temps montre un intérêt bien moindre au $\mathrm{xIx}^{\mathrm{e}}$ siècle qu'au XVIII $^{\mathrm{e}}$ siècle pour la cartographie de la zone ; sans doute parce qu'à cette époque, la ville de Saint-Pierre polarise une grande partie des activités de l'île et que les enjeux militaires sont moindres (fin de la guerre de Sept Ans, fin des guerres de la Révolution et de l'occupation de la Martinique par les Anglais en 1816). Toutes ces caractéristiques confirment la prévalence militaire et nautique des cartes de cette période, et donnent une idée de leurs biais stratégiques et fonctionnels.

Parallèlement à cette cartographie thématique répondant aux besoins de la conquête et de la défense des sites stratégiques, il se développe en France aux XVIII ${ }^{\mathrm{e}}$ et $\mathrm{XIX}^{\mathrm{e}}$ siècles des entreprises de cartographie géométrique, topographique, systématique et à grande échelle, qui commencent à cartographier plus précisément les villes : d'abord la carte de Cassini aux XVII ${ }^{\mathrm{e}}$ et XVIII ${ }^{\mathrm{e}}$ siècles (à l'échelle $1 / 86400$ ), puis au XIX ${ }^{\mathrm{e}}$ siècle le cadastre napoléonien (à l'échelle $1 / 5$ ooo, avec les informations associées : états de sections et liste des parcelles) et la carte d'état-major (1/80 ooo). Bien qu'elles ne soient exemptes ni de biais ni d'erreurs, ces cartes constituent des documents précieux pour les systèmes d'information géographique, historiques et archéologiques, car elles offrent une commune référence sur tout le territoire français, dès le $\mathrm{XVII}^{\mathrm{e}}$ siècle. Or ces couvertures ne sont pas effectuées dans les territoires coloniaux. Il existe donc beaucoup moins de cartes de la Martinique que des espaces comparables de la Métropole, du fait de sa situation coloniale. Une seule carte géométrique et topographique ancienne à grande échelle et couvrant l'ensemble de la Martinique fait référence : la carte de Moreau du Temple, réalisée entre 1770 et 1800 au 1/14 ooo.

Après la destruction de Saint-Pierre au début du $\mathrm{xx}^{\mathrm{e}}$ siècle, beaucoup de familles sont venues accroître les faubourgs déjà existants de Fort-deFrance (quartiers des Terres-Sainville ou de la Cour-Campêche, figure 1) ou s'installer sur des parcelles attribuées pour l'occasion en dehors de la ville, dans le quartier de la Médaille au nord de la commune actuelle, par exemple (Letchimy 1992, 16). Après la Seconde Guerre mondiale, la ville connaît une augmentation démographique massive (elle passe de 60 ooo habitants en 1954 à 100 ooo en 1970), due à la départementalisation ${ }^{4}$, à la centralisation administrative conséquente et à l'exode rural des années 1950-1960. Se développent alors des quartiers populaires encore périurbains, un peu plus lointains, comme Trénelle, Volga ou Texaco - telle une « mangrove urbaine ", selon l'expression d'Aimé Césaire (Letchimy 1992, $7,17)$.

Bien que des lacunes persistent en matière de carte topographique (la première carte topographique de l'IGN dans les années 1950 a été faite non pas au 1/50 ooo comme pour le territoire métropolitain, mais seulement au 1/100 0oo), l'étude des emprises des cartes réalisées au $\mathrm{Xx}^{\mathrm{e}}$ siècle ${ }^{5}$ témoigne d'une diversification des cadrages et d'une focalisation de la cartographie sur les espaces urbains (figure 2). À partir des années 1950, l'évolution de la ville s'accompagne d'une plus importante production de cartes, réalisées à grande échelle (1/5 ooo pour la plupart) par le ministère en charge de la construction (ministère de la Recons- 
truction et de l'Urbanisme puis ministère de la Construction). Néanmoins, l'évolution de la ville se fait alors de manière rapide. Beaucoup de quartiers sont marqués par l'autoconstruction, l'informalité et l'illégalité (30 \% de la population de Fort-de-France est dans ce cas au début des années 1990 [Letchimy 1992, 12]). Or, ces phénomènes très dynamiques sont difficiles à fixer, à quantifier, à délimiter dans l'espace et donc à cartographier sur les cartes topographiques « officielles » de la seconde moitié $\mathrm{du} \mathrm{xx}^{\mathrm{e}}$ siècle. Persiste enfin le problème des toponymes, qui sont quasi exclusivement référencés en français sans qu'apparaisse jamais de graphie créole.

À la fin $d u x^{\mathrm{e}}$ siècle et au début $\mathrm{du} \mathrm{xxI}^{\mathrm{e}}$ siècle, avec la généralisation des systèmes d'information géographique numériques, les représentations cartographiques de la ville de Fort-de-France se normalisent par rapport à celles du reste du territoire français. Sur le Géoportail de l'IGN, on peut consulter les mêmes ressources cartographiques numérisées pour la Martinique que pour le territoire français métropolitain (excepté la carte de Cassini, la carte d'état-major et la carte géologique qui restent absentes). La collectivité territoriale de Martinique s'est dotée, comme la plupart des autres collectivités territoriales françaises, d'une infrastructure de données géographiques (IDG) et d'une plateforme Web, GéoMartinique, où les données ouvertes territoriales sont mises en avant et où de nombreuses données géographiques diverses sur la ville de Fort-deFrance sont consultables. Parallèlement à ces bases de données institutionnelles, se sont développées d'autres bases de données de référence, comme Google Maps et OpenStreetMap (OSM). Toutes ces informations géographiques numériques ne sont plus découpées par les cadres d'un support de papier, elles se multiplient et se diffusent facilement sur le Web grâce à leur format nativement numérique. En un mot, les conditions de la fabrique cartographique ont été considérablement modifiées, et avec elles, le discours cartographique s'est diversifié, les points de vue sur le territoire se sont multipliés et l'autorité cartographique s'est amoindrie (Noucher 2017). La déconstruction critique qu'il était possible de faire des cartes antérieures ne peut plus, en conséquence, se faire exactement de la même manière sur ces informations géographiques contemporaines. Néanmoins, leur utilisation exige toujours une contextualisation critique. Par exemple, OpenStreetMap offre une cartographie intéressante car participative, et donc alternative, notamment concernant les toponymes, dont beaucoup sont renseignés en créole. Néanmoins, cette ressource présente des limites car, étant en perpétuelle construction, la base de données n'est pas exhaustive ni complète ${ }^{6}$. Une autre ressource cartographique intéressante est le fichier de délimitation des IRIS (Îlots regroupés pour l'information statistique). En plus d'être les seules mailles statistiques infracommunales existantes, donc stratégiques pour l'étude quantitative et cartographique d'une ville, les IRIS témoignent d'une délimitation urbaine héritée et révélatrice de " critères géographiques et démographiques et [ont] des contours identifiables sans ambiguïté et stables dans le temps " (INSEE 2016) et ils donnent des informations précieuses de toponymie de quartiers. Néanmoins, se limiter à leur délimitation pour définir les quartiers de la ville serait extrêmement réducteur et fausserait l'analyse (voir la comparaison de la figure 5 et de la figure 6 ci-après), parce qu'ils résultent l'une 
logique avant tout quantitative (ils sont fondés sur un compte démographique d'à peu près 2000 habitants [IRIS200o]) qui ne rend pas entièrement compte de ce qu'est un quartier.

Cette rapide histoire critique de la représentation cartographique de Fort-de-France, mêlant fonction militaire, domination coloniale et développement tardif au $\mathrm{xx}^{\mathrm{e}}$ siècle, révèle la nécessité de mener une contextualisation critique des sources, afin d'évaluer les informations géographiques et historiques qu'il sera possible d'en tirer. À partir de telles sources, l'extraction de données géographiques continues dans le temps et l'espace n'est pas possible, comme ne l'est en général pas l'extraction de données cartographiques parfaitement neutres et objectives. Par conséquent, les systèmes d'information géographique et historique urbains postcoloniaux ne peuvent s'y limiter. Il faut multiplier les sources d'information géographique et historique. Et ce d'autant plus qu'aux conditions de production des cartes s'ajoutent des problématiques liées à leur disponibilité et accessibilité : d'une part, les archives cartographiques de la Martinique sont pour la plupart disséminées en Métropole $^{7}$; d'autre part, peu de ces documents sont numérisés et encore moins accessibles à travers le $\mathrm{Web}^{8}$.

Cet état de la documentation cartographique concernant Fort-deFrance peut être mis en parallèle de problèmes historiographiques plus généraux, fondant les deux principaux courants de théorie littéraire martiniquaise du $\mathrm{xx}^{\mathrm{e}}$ siècle, l'Antillanité d'Édouard Glissant et la Créolité de Jean Bernabé, Raphaël Confiant et Patrick Chamoiseau. Les Antilles souffrent d'un problème historiographique, dû à la situation coloniale et esclavagiste qui a, selon ces auteurs, "raturée " et "stérilisée " la mémoire historique antillaise (Glissant 1981, 133). Pour faire face à cela, les auteurs martiniquais appellent à se servir de la littérature pour notamment " donner à voir les héros insignifiants, les héros anonymes, les oubliés de la chronique coloniale, ceux qui ont mené une résistance tout en détours et en patiences, et qui ne correspondent en rien à l'imagerie des héros occidentalo-français " (Bernabé et al. 1989, 41), et ainsi réécrire l'histoire d'un point de vue créole. Ils appellent à rendre compte de la société par la mise en relation profonde de la multitude et du divers qui la compose, ce qui se traduit concrètement par le multilinguisme, l'importance des détails et l'accumulation d'éléments hétérogènes issus de sources et de discours divers, variés, voire discordants. En suivant ces principes et en puisant dans ces chroniques historiques reconstituées du point de vue créole, il est possible - et même nécessaire - de créer une cartographie historique du territoire martiniquais du point de vue créole, c'est-à-dire de pallier les insuffisances des documents cartographiques martiniquais et de reconstituer des systèmes d'information géohistorique à même de rendre compte de l'évolution complexe de ce territoire. 


\section{Expérimentation de l'intégration d'informations géographiques et historiques littéraires concernant Fort-de- France : méthodologie}

Pour prouver l'efficacité de cette proposition, nous allons nous pencher sur l'intégration des informations géohistoriques sur Fort-deFrance qu'il est possible d'extraire d'œuvres littéraires martiniquaises. Pour cela, nous avons développé une méthodologie en quatre étapes : premièrement, la définition d'un corpus signifiant (bien que pour l'instant limité) ; deuxièmement, la définition, tant sémantique que spatiale, des informations géographiques extractibles ; troisièmement, l'intégration de ces données dans un SIG ; quatrièmement, l'exploration cartographique de ce SIG.

\section{Présentation du corpus}

Plus particulièrement, nous nous sommes appliqués à extraire systématiquement les références géographiques présentes dans deux romans de Patrick Chamoiseau écrits à la fin du $\mathrm{xx}^{\mathrm{e}}$ siècle : Chronique des sept misères, publié en 1986, et Texaco, publié en 1992. Ces deux romans ont été choisis comme premières sources d'analyse dans la perspective méthodologique exposée pour plusieurs raisons. Premièrement, parce que ce sont des romans, donc des productions culturelles (artistiques, subjectives, esthétiques) a priori antagoniques des cartes, donc hautement complémentaires. Deuxièmement, ces sources sont choisies pour leur rayonnement et leur influence dans la culture antillaise contemporaine et dans sa circulation, à la fois du fait de la renommée de leur auteur, $\mathrm{Pa}$ trick Chamoiseau, et de la reconnaissance littéraire dont elles ont bénéficié $^{9}$. Troisièmement, ces deux romans sont emblématiques de l'attention portée à la ville de Fort-de-France dans les romans de la créolité, notamment par le nombre très important de références à l'espace foyalais ${ }^{10}$ (tableau 1).

Le roman Chroniques des sept misères raconte l'histoire des djobeurs du marché de Fort-de-France au milieu du $\mathrm{xx}^{\mathrm{e}}$ siècle. Les djobeurs sont les hommes qui assistent les marchandes dans le transport des marchandises. Chamoiseau décrit ainsi leur activité : «transporter les paniers des marchandes, les produits à exposer sur les nappes de madras, leur ramener une la-monnaie, rendre de menus services en échange de quelques sous, c'était la crème du djob - notre moyen d'existence. " (Chamoiseau $1986,16)$. Le roman raconte plus précisément la vie de Pipi, le plus habile des djobeurs de Fort-de-France, depuis l'installation à Fort-de-France de sa mère, Man Elo, jusqu'à sa mort à la recherche d'un trésor enfoui. Le roman se déroule entre le centre de Fort-de-France où se trouve le marché et les hauteurs de la commune. Létude qui suit concerne la totalité du roman Chronique des sept misères qui, dans l'édition de poche FolioGallimard, fait 288 pages. 
Le roman Texaco raconte l'histoire de la construction du quartier éponyme à l'ouest du centre-ville de Fort-de-France, en entremêlant le récit de Marie-Sophie Laborieux, l'« informatrice ", la " matador-Texaco ", fondatrice et doyenne du quartier, aux paroles rapportées de divers habitants et aux comptes rendus d'acteurs de la ville, dont un urbaniste et le " marqueur de parole " (l'écrivain). Le quartier de Texaco, longtemps informel et fréquemment menacé de destruction par les bulldozers de la mairie jusqu'à sa réhabilitation par le maire Césaire dans les années 1980, tient son nom de l'usine de raffinerie autour de laquelle les premières cases se sont installées. Concernant Texaco, l'étude qui suit se focalise sur trois extraits du roman : les 33 premières pages qui racontent à travers différents points de vue l'arrivée de l'urbaniste dans le quartier de Texaco au début des années 1980 ; 24 pages au centre du roman qui racontent l'arrivée à Fort-de-France des réfugiés de la catastrophe de Saint-Pierre en 1902 ; et les 27 dernières pages du roman, qui reviennent dans le Texaco des années 1980. Chacun de ces extraits correspond à une unité éditoriale (sous-partie du roman). Ces trois extraits représentent un échantillon de 74 pages sur 497 au total, soit à peu près $15 \%$ du livre. Ce prélèvement s'explique par la charge de travail que représente l'extraction manuelle des très nombreuses références géographiques de ce roman (en moyenne, selon cet échantillon, presque six références géographiques par page), et a vocation à guider une future reconnaissance automatique des entités-lieux (voir dernière partie de l'article). 


\section{Définition des informations géographiques et historiques extraites des romans}

TABLEAU 1. DÉCOMPTE DES RÉFÉRENCES À DES LIEUX IDENTIFIABLES DANS LES ROMANS CHRONIQUE DES SEPT MISĖRES ET TEXACO DE PATRICK CHAMOISEAU (DONT LES RÉFÉRENCES À FORT-DE-FRANCE)

\begin{tabular}{|c|c|c|c|c|c|c|c|}
\hline & & \multicolumn{3}{|c|}{$\begin{array}{l}\text { Chronique des } \\
\text { sept misères } \\
(288 \text { p.) }\end{array}$} & \multicolumn{3}{|l|}{$\begin{array}{l}\text { Texaco } \\
\text { (74 p.) }\end{array}$} \\
\hline \multicolumn{2}{|l|}{$\begin{array}{l}\text { RÉFÉRENCES } \\
\text { À DES LIEUX }\end{array}$} & Nombre & $\begin{array}{l}\text { Nombre } \\
\text { par } \\
\text { page }\end{array}$ & $\%$ & Nombre & $\begin{array}{l}\text { Nombre } \\
\text { par } \\
\text { page }\end{array}$ & $\%$ \\
\hline \multirow{3}{*}{$\begin{array}{l}\text { Échelle infracom- } \\
\text { munale } \\
\text { [4] }\end{array}$} & Total [1] & 176 & 0,61 & 60,69 & 260 & 3,51 & 60,05 \\
\hline & $\begin{array}{l}\text { Information } \\
\text { supplémentaire sur } \\
\text { le lieu } \\
{[2]}\end{array}$ & 82 & 0,28 & 28,28 & 72 & 0,97 & 16,63 \\
\hline & $\begin{array}{l}\text { Désignation } \\
\text { par un } \\
\text { toponyme } \\
{[3]}\end{array}$ & 136 & 0,47 & 46,90 & 152 & 2,05 & 35,10 \\
\hline \multirow{3}{*}{$\begin{array}{l}\text { - Dont } \\
\text { Fort-de-France } \\
{[4]}\end{array}$} & Total [1] & 144 & 0,5 & 49,66 & 237 & 3,2 & 54,73 \\
\hline & $\begin{array}{l}\text { Information } \\
\text { supplémentaire sur } \\
\text { le lieu } \\
{[2]}\end{array}$ & 74 & 0,26 & 25,52 & 68 & 0,92 & 63,55 \\
\hline & $\begin{array}{l}\text { Désignation } \\
\text { par un } \\
\text { toponyme } \\
{[3]}\end{array}$ & 100 & 0,35 & 34,48 & 134 & 1,81 & 55,83 \\
\hline \multirow{3}{*}{$\begin{array}{l}\text { Échelle } \\
\text { communale } \\
{[5]}\end{array}$} & Total [1] & 64 & 0,22 & 22,07 & 128 & 1,73 & 29,56 \\
\hline & $\begin{array}{l}\text { Information } \\
\text { supplémentaire sur } \\
\text { le lieu } \\
{[2]}\end{array}$ & 16 & 0,06 & 5,52 & 33 & 0,45 & 7,62 \\
\hline & $\begin{array}{l}\text { Désignation } \\
\text { par un } \\
\text { toponyme } \\
{[3]}\end{array}$ & 54 & 0,19 & 18,62 & 57 & 0,77 & 13,16 \\
\hline \multirow{3}{*}{$\begin{array}{l}\text { Échelle } \\
\text { supérieure } \\
{[6]}\end{array}$} & Total [1] & 50 & $\mathbf{0 , 1 7}$ & 17,24 & 45 & 0,61 & 10,39 \\
\hline & $\begin{array}{l}\text { Information } \\
\text { supplémentaire sur } \\
\text { le lieu } \\
\text { [2] }\end{array}$ & 9 & 0,03 & 3,10 & 9 & 0,12 & 2,08 \\
\hline & $\begin{array}{l}\text { Désignation } \\
\text { par un } \\
\text { toponyme } \\
{[3]}\end{array}$ & 50 & 0,17 & 17,24 & 38 & 0,51 & 8,78 \\
\hline \multirow{3}{*}{$\begin{array}{l}\text { Toutes } \\
\text { références } \\
\text { localisables }\end{array}$} & Total [1] & 290 & 1,01 & 100,00 & 433 & 5,85 & 100,00 \\
\hline & $\begin{array}{l}\text { Information } \\
\text { supplémentaire sur } \\
\text { le lieu } \\
{[2]}\end{array}$ & 107 & 0,37 & 36,90 & 114 & 1,54 & 26,33 \\
\hline & $\begin{array}{l}\text { Désignation } \\
\text { par un } \\
\text { toponyme } \\
\text { [3] }\end{array}$ & 240 & 0,83 & 82,76 & 247 & 3,34 & 57,04 \\
\hline
\end{tabular}

Les numéros entre crochets renvoient à l'explication des catégories dans le texte. Total des références extraites : 723 . 
Nous avons extrait des informations géographiques et historiques de ce corpus (synthèse dans le tableau 1) en relevant à la lecture des romans, exhaustivement et systématiquement, chaque citation d'un lieu non ambigu et qu'il était possible de localiser dans l'espace géographique réel (catégorie [1]). Ces informations géohistoriques sont modélisées comme la combinaison de deux groupes d'informations : d'une part, les informations concernant leurs conditions d'apparition dans le texte (la citation à proprement parler), et d'autre part, les informations concernant le lieu auquel elles font référence - plusieurs citations différentes pouvant faire référence à un même lieu.

Concernant le premier volet - les conditions d'apparition dans le texte des informations géohistoriques -, plusieurs types d'informations sémantiques sont associés aux citations pendant le relevé manuel (qu'il serait très difficile d'automatiser) :

- Le premier type d'informations concerne la date ou la période à laquelle renvoie la citation, qui est définie selon son contexte diégétique.

- Le deuxième type d'informations concerne les modalités de la citation : est-elle faite par un toponyme (par exemple, «Fort-deFrance ", " Texaco ", " rue Lamartine », catégorie [3]), ou indirectement par un groupe nominal ou prépositionnel (par exemple, "chez elle », " derrière ma case », " derrière le temple adventiste », etc.) ? Est-ce une désignation directe ou relative (par exemple, «À proximité des falaises de Cases-Pilote ", Texaco, p. 24)?

- Le troisième type d'informations concerne la contextualisation documentaire de la citation, autrement dit la situation de la citation au sein de la source dont elle est extraite, en l'occurrence le roman (page, section éditoriale, identité du narrateur, etc.).

- Enfin, le quatrième type d'information concerne la qualité géographique de la citation : quel type de phénomène géographique cette référence désigne-t-elle ? Désigne-t-elle une position fixe dans l'espace (par exemple, " les habitants de Texaco", Texaco, p. 2o) ou un déplacement (par exemple, "Nous avions couru de Saint-Pierre ", Texaco, p. 207) ? Est-ce simplement la localisation d'un événement romanesque, ou donne-t-elle une information géographique supplémentaire sur le lieu qu'elle désigne ? Ce qui est le cas par exemple de la citation suivante, dans laquelle le quartier de Texaco fait l'objet d'une description que nous qualifions d' "informative » : « Nous obtînmes l'eau courante à Texaco. Les services de la mairie vinrent, en plus, nous déverser des caillasses sur la boue, nous cimenter nos routes, dresser des escaliers qui sillonnaient les pentes en épousant nos traces " (Chamoiseau 1992, 471). C’est également le cas dans cet autre exemple qui donne des informations en matière d'agencement spatial : « il longea le marché aux légumes vers la rue Isambert. Cela lui prit quatre secondes, vite rattrapées en remontant à grand allant la rue Isambert, dégagée à cette heure du samedi. Dépassant la cour Perrinon, traversant la rue Victor-Sévère, il dévala la ruelle Abbé-Lecornu absolument déserte, et déboucha sur le boulevard du Général de Gaulle, encombré mais large comme un nez, donc sans problème pour un maître de la brouette » (Chamoiseau 
1986, 90-91). Dans ces cas, les citations sont qualifiées de citations localisables avec information sémantique supplémentaire sur le lieu (catégorie [2]).

Létude statistique des citations dénombrées dans le tableau montre la grande qualité de l'information géohistorique sur la ville de Fort-deFrance qu'il est possible de tirer des extraits des deux romans étudiés : toutes les cinq pages en moyenne dans Chronique des sept misères (0,26 citation par page), il y a une citation localisable désignant un lieu à l'intérieur de Fort-de-France et accompagnée d'une information géographique et sémantique riche. Et, dans l'extrait de Texaco, il y en a quasiment une par page en moyenne $(0,92)$. Les références à Fort-deFrance représentent à peu près la moitié des citations localisables dans chacun des romans. La synthèse statistique montre également la masse de citations localisables (723 au total), leur précision et leur diversité géographique, et donc l'efficacité probable du recours à la cartographie pour leur exploration.

Dans notre modélisation des informations géohistoriques extraites des romans martiniquais, ces nombreuses citations sont reliées à une liste de lieux auxquels elles font référence. Ces lieux sont caractérisés de diverses manières, notamment par des informations d'échelle, ce qui permet de savoir combien de références renvoient à des lieux d'échelle infracommunale (catégorie [4] du tableau), communale (catégorie [5]) ou supérieure, c'est-à-dire équivalente à plusieurs communes, un département, une région administrative (ou équivalent), un pays, ou une région du monde (catégorie [6]). Ces trois catégories d'échelles ont été construites selon l'emprise de notre étude, à savoir Fort-de-France. La première catégorie sera particulièrement importante pour comprendre les disparités de représentation des lieux à l'intérieur de la ville (voir les cartes ci-dessous). 


\section{Modélisation spatiale et construction d'un SIG pour l'exploration spatiale des informations géohistoriques romanesques}

FIGURE 3. SCHÉMA CONCEPTUEL DU SIG CONSTITUÉ POUR L’ÉTUDE DES INFORMATIONS GÉOHISTORIQUES EXTRACTIBLES DE ROMANS MARTINIQUAIS SUR LA VILLE DE FORT-DE-FRANCE

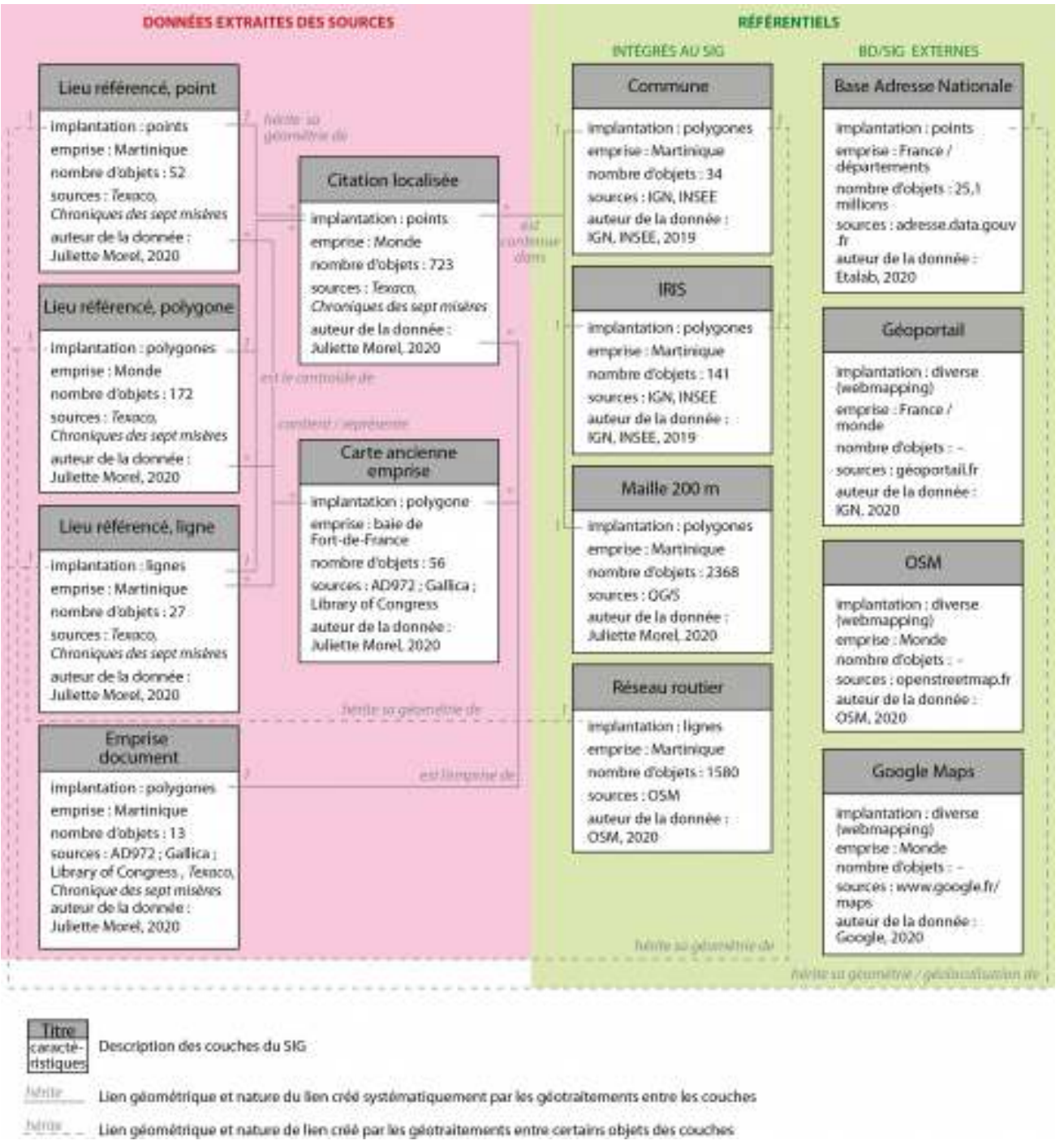

État du schéma conceptuel en octobre 2020

Image produite par l'autrice

Ces lieux constituent les premières couches d'informations géographiques du SIG résultant (voir tables de gauche sur la figure 3) et sont répartis selon l'implantation (point, polygone ou ligne) qui convient le mieux à leur représentation cartographique - nous avons fixé le degré de schématisation conceptuelle de l'implantation selon notre échelle de référence, celle de la ville. Par exemple, les lieux Fort-de-France, Texaco, centre-ville ou France auront une implantation polygonale, la maison d'Aimé Césaire, l'hôtel de ville ou la case de la narratrice de Texaco auront une implantation ponctuelle, et le boulevard du Général de Gaulle aura une implantation linéaire.

Pour géolocaliser les lieux situés en France, nous sommes passés par une première étape de géocodage avec l'outil de géocodage du gouvernement français fondé sur la base adresse nationale (BAN $\left.{ }^{11}\right)$, que nous avons ensuite repris à la main, pour trois principales raisons : 
- D’abord parce qu'après analyse des résultats du géocodage, nous avons jugé que 36,6\% d'entre eux étaient incorrects et que 6,3\% nétaient pas assez précis. Pour corriger cela, nous avons croisé plusieurs sources : les cartes anciennes citées, des référentiels cartographiques comme les cartes IGN présentes sur Géoportail, Google Maps, OpenStreetMap, la toponymie des IRIS, la toponymie du réseau de transport public (voir partie sur fond vert de la figure 3), la connaissance personnelle du terrain, ou encore nous avons recoupé entre elles les informations géographiques littéraires.

- Ensuite parce que le géocodage crée des points (géométriquement définis par une longitude et une latitude) et qu'il fallait par conséquent ensuite rapporter les lieux dont l'implantation avait été définie comme ligne ou polygone aux entités géométriques correspondantes. Pour cela, nous nous sommes rapportés à des couches d'information géographique de référence : les limites des communes et des IRIS produites par l'IGN et le réseau viaire d'OpenStreetMap (voir partie sur fond vert de la figure 3).

- Enfin parce que les lieux hors de France ne sont pas référencés dans la base de données BAN. Pour localiser ces lieux, nous avons principalement utilisé « manuellement » OpenStreetMap et Google Maps.

Une fois tout cela fixé, demeurait le problème de l'inégale précision de la localisation des lieux romanesques (nous laissons de côté la question des lieux imaginaires). Par exemple, le lieu correspondant à la case de Marie-Sophie Laborieux, la narratrice de Texaco (cité trois fois dans les extraits étudiés du roman), devrait, selon les choix méthodologiques qui viennent d'être exposés, être défini comme un point. Or, sa localisation précise n'est pas connue, on sait seulement que la case se situe quelque part dans le quartier de Texaco. Pour résoudre ce problème, nous avons choisi de conserver l'implantation à laquelle serait modélisé le lieu si sa localisation était connue avec précision - ici par exemple, le point -, de fixer sa localisation par rapport à l'entité géographique connue avec précision d'échelle supérieure la plus proche - ici, par rapport au quartier Texaco - et de renseigner le degré de précision de la localisation dans les attributs du lieu - ici, un degré de précision faible. Dans le cas d'une implantation ponctuelle, on stocke le centroïde de la géométrie polygonale ou linéaire de l'entité connue d'échelle supérieure la plus proche : dans notre exemple, on modélisera donc la case de Marie-Sophie Laborieux par un point situé au centroïde du polygone délimitant le quartier Texaco, tout en caractérisant la précision spatiale de faible.

Ces différentes étapes permettent d'associer une géométrie localisée à tous les lieux auxquels renvoient les citations localisables des romans. Cependant, toutes ces géométries ne sont pas de même nature - n'ayant pas toutes la même échelle, la même précision, ni la même implantation - et sont donc topologiquement difficiles à comparer. C'est pourquoi nous avons choisi de doubler ces couches de lieux d'une couche regroupant toutes les citations localisées, auxquelles nous avons attribué une implantation ponctuelle (voir le centre de la figure 3). Pour cela, les citations faisant référence à des lieux dont l'implantation est déjà ponctuelle héritent directement de leur géométrie, et les citations faisant référence à des lieux dont l'implantation est polygonale ou linéaire héritent des centroïdes de leur géométrie, ainsi que de l'information d'échelle et de précision associée au lieu. Les représentations cartogra- 
phiques qui suivent ont pour objectif d'explorer précisément cette couche de données, afin de visualiser la spatialité des références géographiques figurant dans les romans, et de les comparer à des informations géographiques extraites d'autres sources comme les cartes anciennes, et à des informations géographiques dites " de référence " (ou " référentiels » dans la partie sur fond vert de la figure 3). Pour pallier le biais géométrique imposé par l'implantation ponctuelle uniformisante (parfois abusivement), nous doublons à chaque fois les représentations ponctuelles de représentations par densité (elle-même à plusieurs échelles, et par rapport aux différents référentiels, voir figure 5 et figure 7) (Moncla, Gaio et Joliveau 2018).

\section{Exploration cartographique des informations géohistoriques romanesques}

Une fois construit ce système d'information géographique intégrant les citations localisables extraites de romans martiniquais, il fut possible d'en explorer la spatialité par la cartographie. Ces explorations montrent les disparités de représentations des différents espaces de la ville, à la fois selon la source considérée, la période historique visée et la qualité de l’information recherchée. 
FIGURE 4. LOCALISATION DES LIEUX RÉFÉRENCÉS DANS CHRONIQUE DES SEPT MISĖRES ET TEXACO

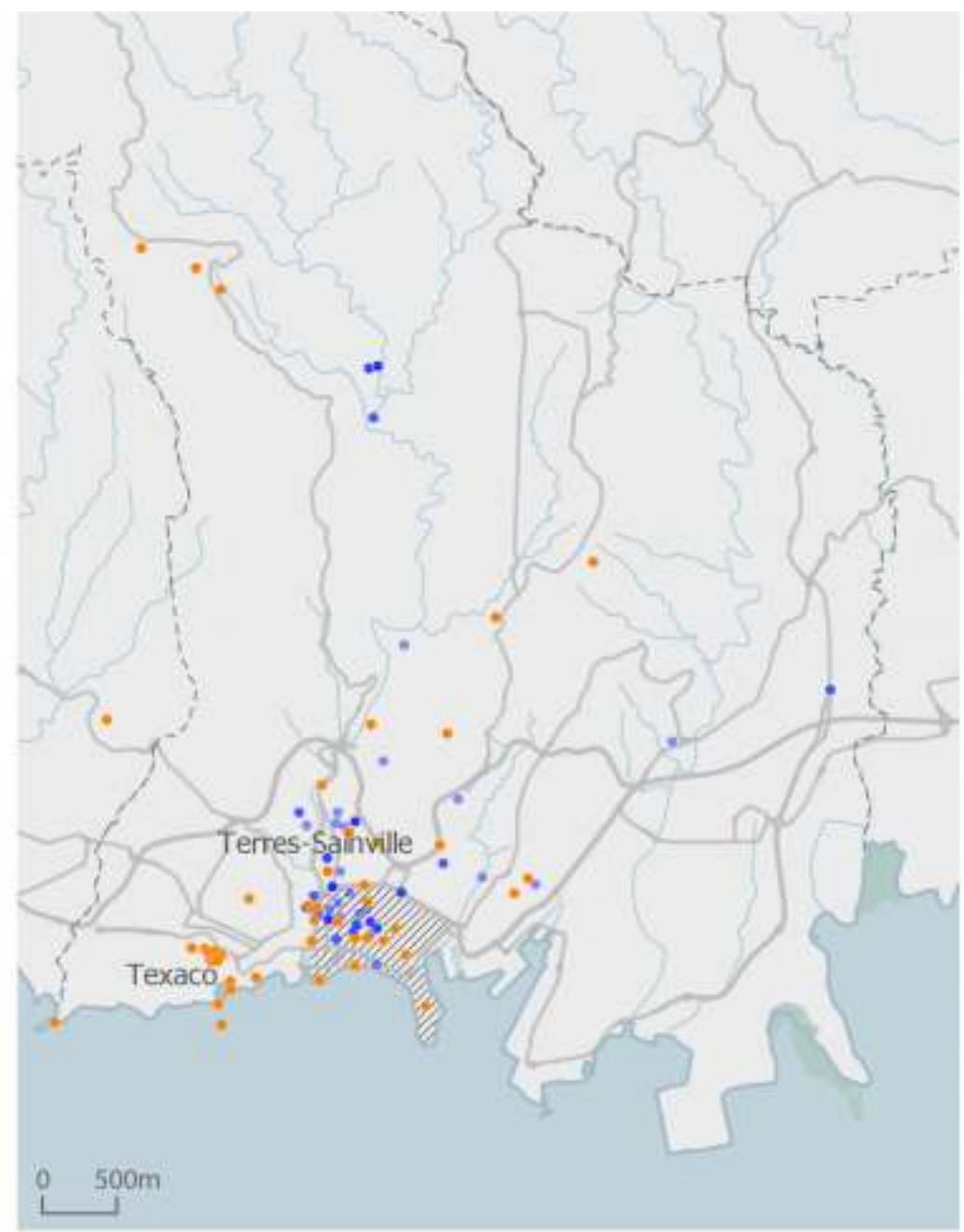

- Localisation des références géographiques citées dans Chronique des sept misères

- Localisation des références géographiques citées dans Texaco

.... Limites de commune en 2020

— routes principales en 2020

réseau hydrographique

Centre ville historique de Fort-de-France

Image produite par l'autrice 


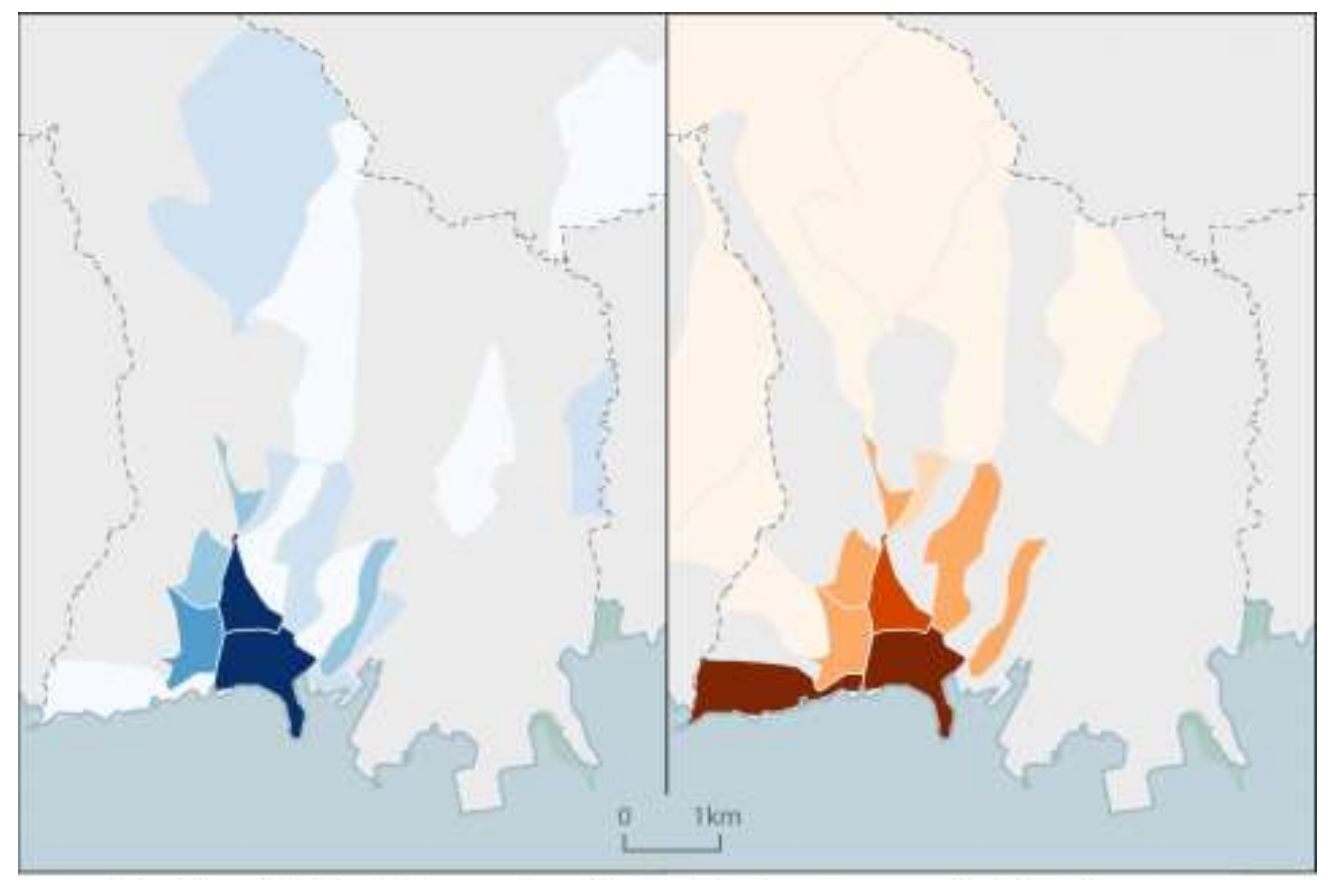

Densitẻ par IRIS des références géographiques citèes dans les romans de Patrick Chamoiseau (en nombre de citations par $\mathrm{km} 2$; discrétisation par progression algorithmique ; zoom sur la ville de Fort-de-France et sur les lieux d’échelle infra-communale)

Chronique des sept misères

Texaco

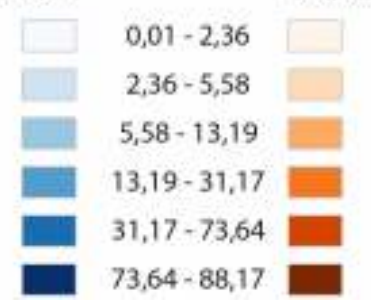

Image produite par l'autrice

On peut remarquer, sur la cartographie différenciant les citations localisables extraites de Chronique des sept misères et celles extraites de Texaco, les similitudes et les différences de leur localisation dans les deux romans. En termes de similitudes, on remarque l'importance du centre-ville et des quartiers immédiatement périphériques, notamment du quartier des Terres-Sainville au nord-ouest du centre, le plus ancien faubourg populaire de Fort-de-France. En termes de différences, on remarque une forte concentration des références géographiques dans le quartier de Texaco sur la côte à l'ouest du centre-ville dans le roman du même nom, et une forte concentration autour des trois marchés de Fortde-France, au nord-ouest à l'intérieur du centre-ville, dans Chronique des sept misères. Cette polarisation autour des marchés et de la Croix-Mission, carrefour où arrivent les routes venant des alentours de la ville, définit un nouvel ensemble géographique cohérent, qui englobe le nordouest du centre de Fort-de-France, l'est du quartier de la Cour Campêche et le sud du quartier des Terres-Sainville. Cet espace chevauche les frontières des quartiers définis par les sources de l'information géographique officielle, que ce soit la délimitation des IRIS de l'INSEE-IGN ou les cartes plus ou moins anciennes, qui représentent la plupart du temps sur des feuillets séparés le centre et les faubourgs périphériques (figure 6). C'est pourquoi l'agrégation par IRIS n'est pas la plus à même de résumer la répartition des références géographiques littéraires, et que 
nous les remplacerons dans les cartes suivantes par des carreaux de $200 \mathrm{~m}$ de côté faisant apparaître des variations à une échelle plus fine, tout en respectant le principe d'imprécision des références ponctuelles.

FIGURE 6. COMPARAISON DES RÉFÉRENCES GÉOGRAPHIQUES CITÉES DANS LE ROMAN CHRONIQUE DES SEPT MISĖRES ET DES DÉLIMITATIONS SPATIALES DÉFINIES DANS D'AUTRES SOURCES D'INFORMATION GÉOGRAPHIQUE

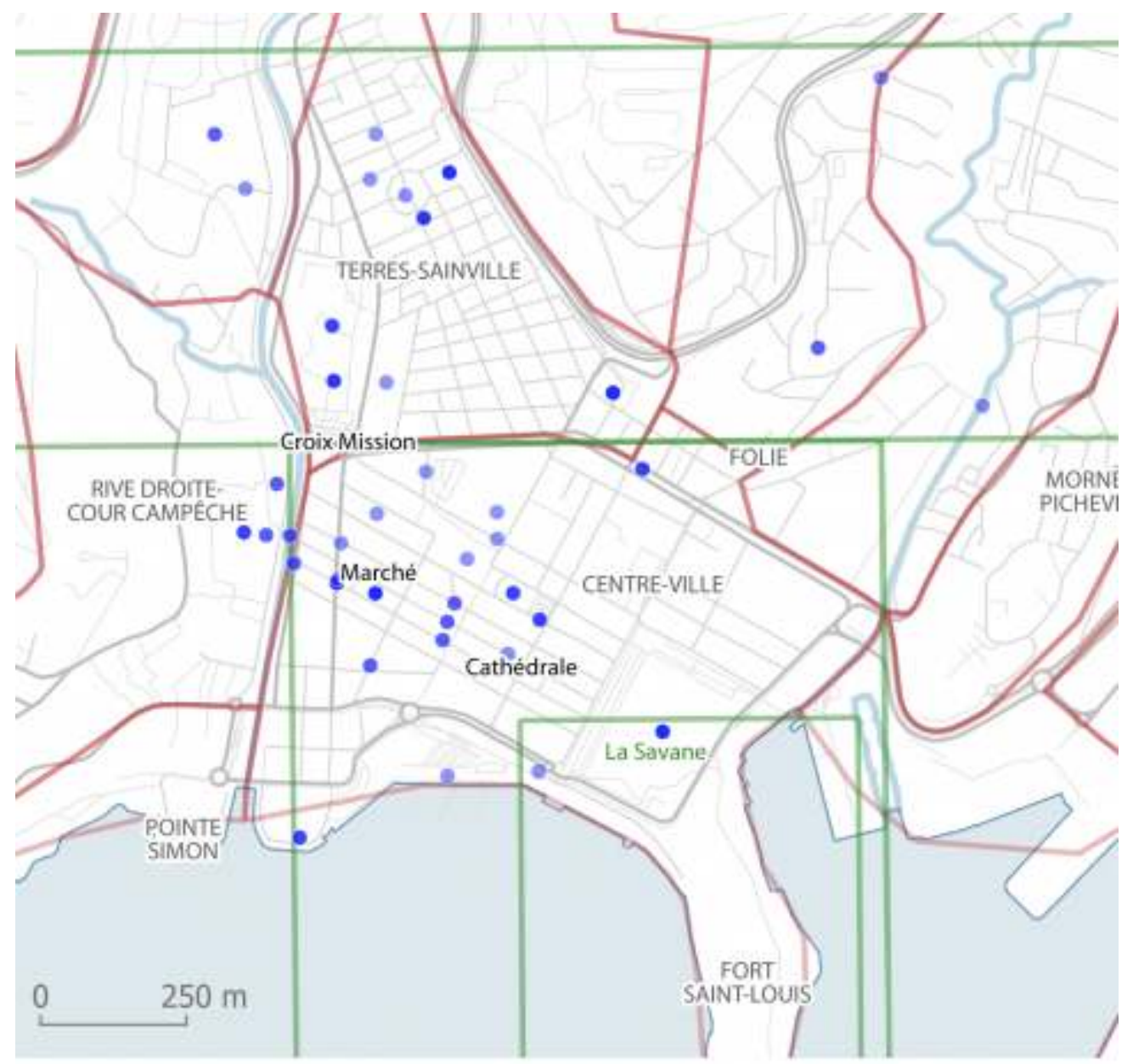

- Références géographiques citées

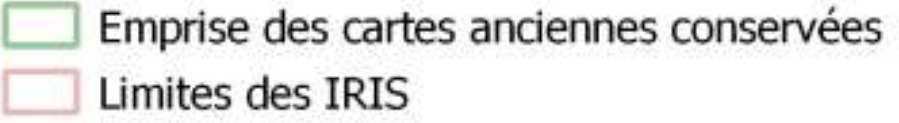

Image produite par l'autrice

L’observation des différentes époques auxquelles les références géographiques renvoient fait apparaître des logiques historiques. Sur les deux figures ci-dessous (figure 7 et figure 8), on voit l'étalement urbain à travers le temps, des lieux renvoyant à des périodes de référence antérieures à 1900, peu nombreux et concentrés dans le centre historique, aux lieux renvoyant au $\mathrm{xx}^{\mathrm{e}}$ siècle, beaucoup plus nombreux et diversifiés. $\mathrm{Au}$ début $\mathrm{du} \mathrm{xx}^{\mathrm{e}}$ siècle, le faubourg populaire de Terres-Sainville qui jouxte le centre-ville au nord-ouest est déjà implanté. C'est un des endroits où se sont réfugiés les rescapés de la catastrophe de Saint-Pierre après 1902, à l'image du personnage d'Esternome dans Texaco. Il est un des pôles géographiques importants des deux romans. Beaucoup de références géographiques renvoyant à la seconde moitié $\mathrm{du} \mathrm{xx}^{\mathrm{e}}$ siècle (entre 1947 et 1990) se situent dans le quartier de Texaco, qui se développe de manière informelle à partir des années 1950, mais n'est réhabilité par la mairie de Fort-de-France que dans les années 1980. Cette très 
forte polarisation est un biais des sources, dû à l'importance de ce quartier dans le roman du même nom. Néanmoins, elle est révélatrice du développement des quartiers informels plus périphériques et de leur connexion progressive au centre-ville. D'autres quartiers de ce type sont cités, comme le quartier du Morne Pichevin à l'opposé est du centre-ville. Elle est également révélatrice de la quantité d'informations substantielle que le roman peut fournir à propos du développement de quartiers dans la seconde moitié $\mathrm{du} \mathrm{xx}^{\mathrm{e}}$ siècle.

Il est également intéressant de noter la quasi-absence de données concernant la ville de Fort-de-France au XIX ${ }^{\mathrm{e}}$ siècle. Si on observe la Martinique entière, on constate que les références géographiques renvoyant au $\mathrm{XIX}^{\mathrm{e}}$ siècle concernent majoritairement la ville de Saint-Pierre (37 références sur 42), ce qui rend compte du réseau urbain de la Martinique au $\mathrm{XIX}^{\mathrm{e}}$ siècle, dominé par cette dernière, alors capitale économique, commerciale et culturelle des Antilles francophones.

FIGURE 7. DENSITÉ DES RÉFÉRENCES GÉOGRAPHIQUES CITÉES DANS CHRONIQUE DES SEPT MISĖRES ET TEXACO SELON L'ÉPOQUE DE RÉFÉRENCE. ZOOM SUR LA VILLE DE FORT-DE-FRANCE

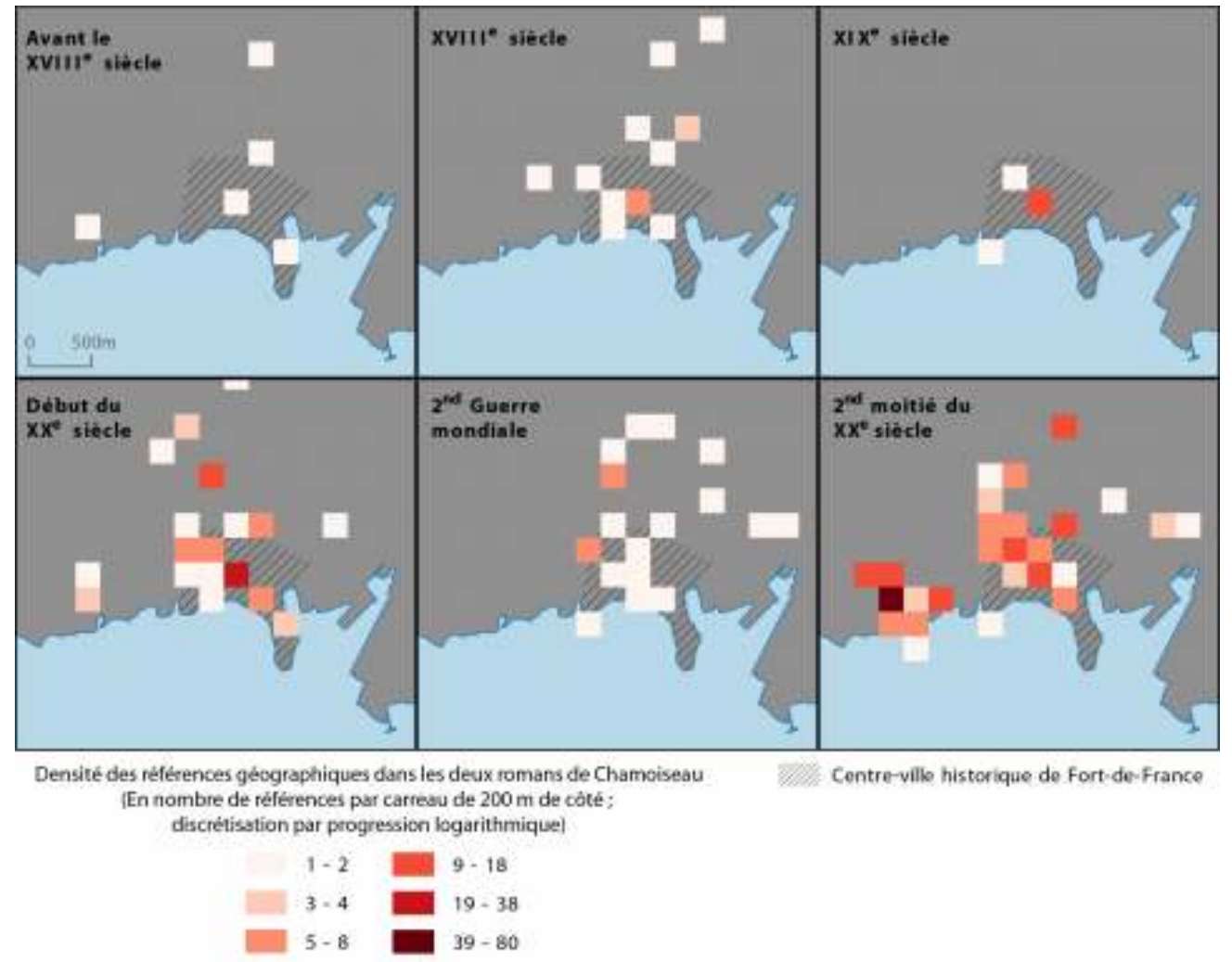

Image produite par l'autrice 


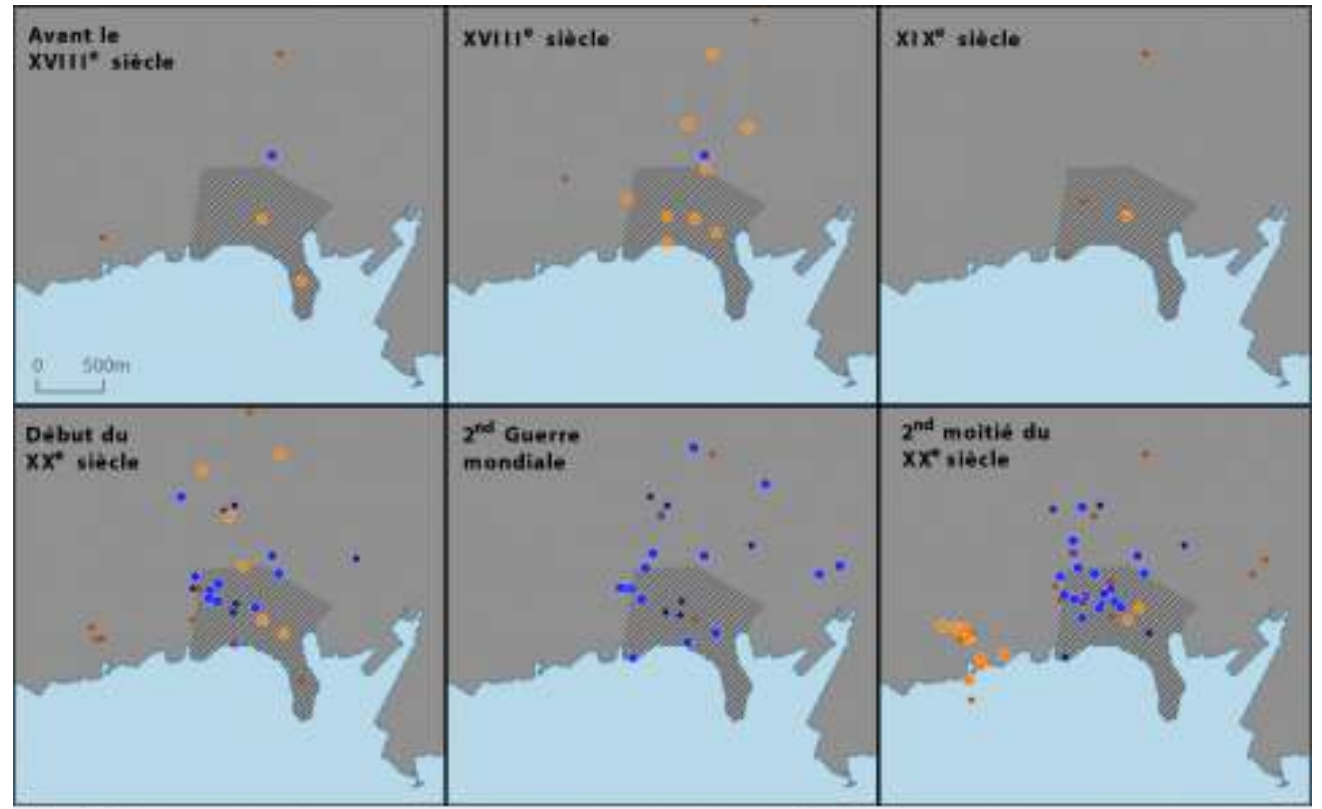

- References géographiques, avec information supplementaire citées dans Chronique des septs miseres Références géographiques, sans information supplémentaire, citees dans Chvonique des seppts misères Références géographiques, awec information supplèmentaire, citèes dans Texaco Réferences géographiques, sans information supplémentaire, citées dans texoco Centre-ville historique de Fort de France

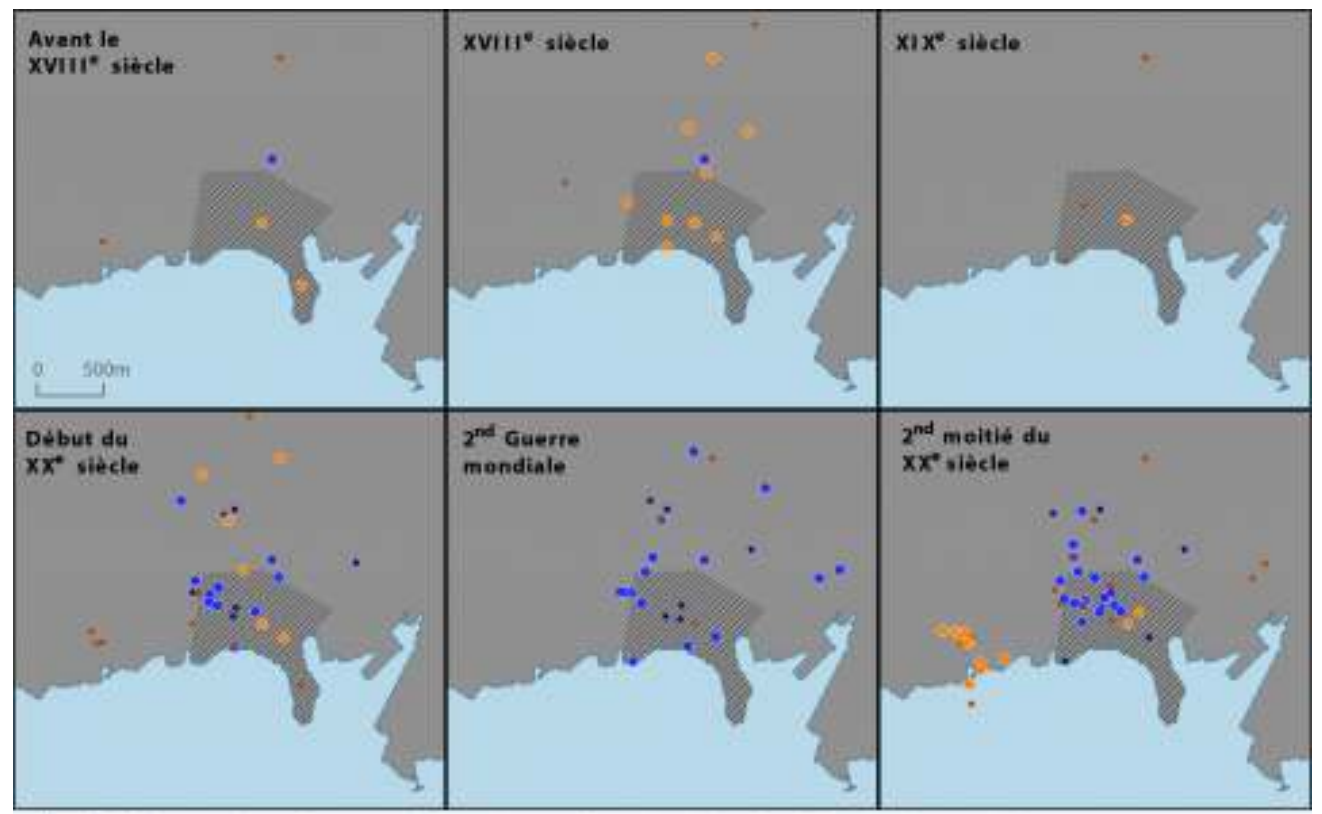

- References géographiques, anec information stupplémentaire citées dans Chronique des septs miseres Références géographiques, sans information supplémentaire, citées dans Chvoniquze des sepes misères Références gebographiques, amoc information supplèmentaire, citees dans Texaco Réferences géographiques, sans information supplementaire, citees dans texoco Centre-ville historique de Fort de -France

Image produite par l'autrice

Enfin, en vue de leur utilisation géohistorique dans un cadre dépassant celui des romans, il est important de mettre en perspective ces répartitions dans l'espace et dans le temps avec la qualité de l'information géographique et historique recueillie (figure 8). Cette cartographie confirme les différents constats faits à partir des autres cartes : redéfinition de la cohérence de certains ensembles infra-urbains, apparition 
d'un quartier commercial au nord-ouest du centre-ville, répartition différente des informations géographiques dans le temps, extension des limites de la ville - et donc des informations sur celles-ci - entre la fondation du Fort-Royal à la fin des années 1630 et les années 1990. Elle confirme également l'intérêt des informations géographiques extraites des romans, puisque, en plus de pouvoir y suivre l'histoire de la ville, elles promettent d'y apporter une contribution, notamment par les nombreuses informations substantielles concernant les quartiers populaires qui y sont données.

\section{Résultats et perspectives : accumuler et comparer pour une cartographie complexe des espaces (post-)colonisés}

\section{Résultats}

L'étude comparée des sources cartographiques et des références géographiques et historiques littéraires sur Fort-de-France démontre plusieurs choses. Premièrement, elle montre, non seulement la nécessité politique de l'intégration de sources d'informations géographiques et historiques alternatives, comme les romans, mais aussi la richesse du recours à ces documents souvent laissés de côté pour reconstruire la géographie historique d'une ville.

Deuxièmement, l'exploration cartographique est une méthode efficace pour la comparaison des sources de l'information géographique dans une perspective critique. Elle permet de recenser et de faire apparaître concrètement les effets de la spécialisation et de la partialité des sources, qui dessinent par conséquent des géographies différentes. Il est important de rappeler à cet égard que cette étude propose une exploration cartographique de sources historiques variées, dans le but d'appréhender spatialement et dans son ensemble une multitude complexe de données (les emprises des nombreuses cartes anciennes, les 723 références géographiques localisables extraites des romans de Patrick Chamoiseau) ; mais qu'elle n'a pas l'ambition de remplacer une géographie fine des représentations, une monographie d'histoire urbaine sur Fortde-France, ni l'analyse narratologique des romans de Chamoiseau. Au contraire, ces différentes démarches sont complémentaires et à connecter : la cartographie est efficace pour le recensement spatiotemporel et peut aider à s'orienter dans un corpus important de sources et d'espaces en vue de leurs analyses plus fines, qu'elles soient géographiques, historiques ou littéraires.

Troisièmement, il découle directement des deux premières conclusions que cette démarche n'est valide qu'avec la perspective d'une accumulation de sources et d'informations géographiques hétérogènes pour construire des systèmes d'information géohistorique plus complets et plus complexes. Dans cette perspective, il ne suffit en effet pas d'extraire les références géographiques d'un ou deux romans - même si ce sont des centaines - pour compléter la représentation biaisée d'une carte ancienne. Il faut le plus de données possible issues de sources les plus variées possible : davantage de cartes anciennes, des données en série, des 
sources textuelles, littéraires, administratives, judiciaires, picturales, etc. En ce sens, l'étude présentée ici n'est qu'exploratoire et préliminaire à une telle accumulation.

Enfin, le quatrième enseignement s'inscrit dans une perspective résolument constructiviste : il est abusif de parler de "système d'information géographique et historique » dans l'absolu, ceux-ci étant avant tout des systèmes d'information documentaire, rendant d'abord compte des sources et représentations disponibles et numérisables et non de la ville elle-même.

\section{Pistes et perspectives méthodologiques}

Chacune de ces conclusions positives soulève de nouvelles questions méthodologiques auxquelles il sera impératif de répondre pour achever notre méthodologie, et qui constituent autant de pistes de prolongement. Maintenant que nous avons prouvé qu'il était intéressant de considérer les informations géohistoriques extractibles de sources alternatives rarement exploitées, une question centrale se pose : comment faire concrètement, c'est-à-dire comment extraire, accumuler, intégrer et croiser des informations géographiques hétérogènes ? Car cette démarche entraîne des problèmes considérables tant d'ordre conceptuel en matière de définition et d'analyse de corpus - que pratique - en matière d'extraction, de gestion, de comparaison et de croisement de la masse des informations hétérogènes induites.

Du côté théorique, il faudra poursuivre le recensement et l'analyse critique fine de toutes les sources disponibles (séries statistiques, sources textuelles diverses, sources iconographiques, etc.), comme nous l'avons fait sur les cartes anciennes.

D'un point de vue méthodologique, il faudra continuer à explorer l'immense problème de la modélisation des informations, c'est-à-dire la manière dont elles sont décrites, qui induit par extension la manière dont elles seront implémentées informatiquement et vectorisées cartographiquement, et la manière dont elles seront croisées et interrogées. Concernant par exemple la dimension spatiale de cette question, il faudra complexifier la modélisation, à travers un travail minutieux sur la vectorisation cartographique des sources : car référencer l'emprise d'une carte ancienne donne de premières informations, comme nous l'avons montré, mais ne suffit pas à rendre compte de ce que la carte représente vraiment et comment. Il faudra également référencer les informations données par la carte (que celle-ci soit une information statistique, de localisation, de liaison, etc.), mais aussi les toponymes et les espaces effectivement dessinés, les implantations et les variables visuelles utilisées.

Plus généralement, il faudra sans cesse avoir en tête qu'il est absolument nécessaire de réfléchir à l'incidence de la modélisation construite sur les croisements et les résultats géographiques et historiques qui pourront en être tirés. À cet égard, une piste est, nous semble-t-il, à privilégier : sortir des modèles classiques des systèmes d'information géographique, organisés autour de la mise en relation de " couches ", fichiers réunissant des entités géographiques de même nature et de même implantation, comme nous l'avons fait (une couche pour les communes, une couche pour le réseau viaire, une couche pour les IRIS, une couche pour les citations localisables, etc., voir figure 3). Ces modèles, 
sur lesquels se fondent tous les logiciels de SIG, sont limités dans le cas des systèmes d'information cumulatifs, parce que, même s'ils permettent de croiser toutes les informations spatialement, ils ne permettent pas de croisements temporels ni sémantiques complexes entre couches, et parce qu'il est rapidement peu optimal d'accumuler de nombreuses couches d'informations. Il faut substituer à cela des systèmes d'information spatiotemporels et documentaires permettant la manipulation plus souple des informations. L'atomisation de l'information (par exemple, telle qu'elle est pensée dans la modélisation archéologique OH_FET [Rodier et Saligny 2010]), sa description et son stockage de manière multidimensionnelle (comme dans la triade espace-temps-sémantique [Peuquet 1994]), qui constituent des principes de modélisation spatiotemporelle devenus classiques en histoire ou en archéologie, offrent des pistes intéressantes pour décrire et donc ensuite appréhender et croiser les informations autant de manières spatiale, temporelle, sémantique que documentaire (Morel, Crouzevialle et Massoni 2020) (figure 9).

FIGURE 9. MODÈLE CONCEPTUEL SIMPLIFIÉ D'UN SYSTÈME D'INFORMATION RÉELLEMENT GÉOGRAPHIQUE, HISTORIQUE ET DOCUMENTAIRE, FONDÉ SUR LE PRINCIPE D’ATOMISATION ET DE MULTIDIMENSIONNALITÉ DE L'INFORMATION

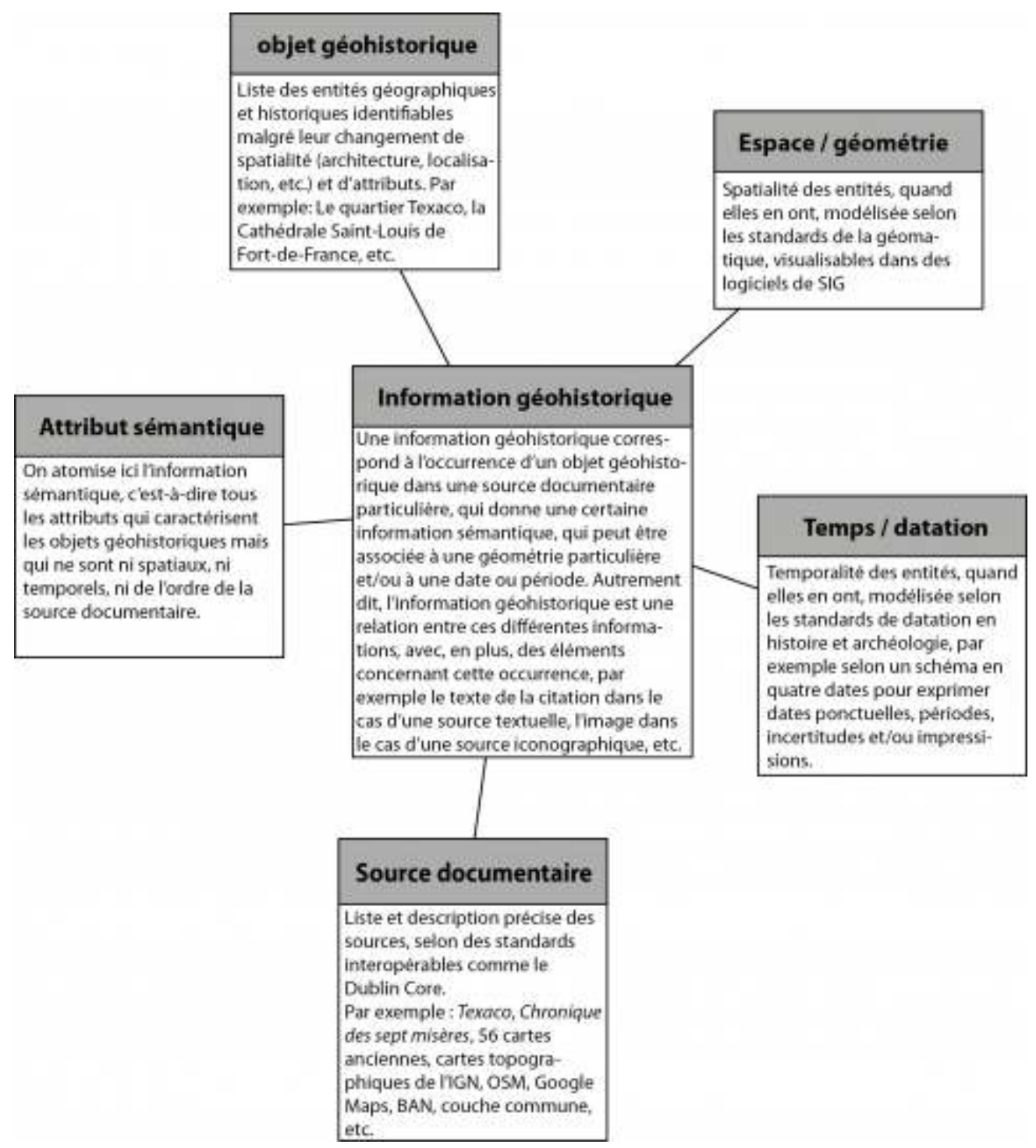

Image produite par l'autrice 
Pour parfaire la modélisation, il faudra également se confronter à l'enjeu majeur de la qualité des données géohistoriques (Devillers et Jeansoulin 2005). Il n'est en effet pas question de considérer qu'une information issue d'un roman fictionnel a le même statut qu'une information issue d'une étude statistique, d'une carte topographique de référence ou d'une enquête scientifique. Notre démarche implique une réflexion minutieuse sur la question de la valeur informationnelle d'une donnée ; et il sera nécessaire d'intégrer à la modélisation les résultats du travail critique mené sur chacune des sources considérées.

Enfin, d'un point de vue davantage technique, il faudra utiliser ou construire des outils facilitant l'extraction d'informations, tout en respectant les principes conceptuels et de modélisation décrits jusqu'ici ; car une extraction manuelle des informations géohistoriques ne permettra pas la constitution de jeux suffisamment grands pour devenir significatifs. Deux directions s'offrent alors : la première est l'automatisation de l'extraction des informations géohistoriques, en suivant les recherches en cours notamment dans le domaine de la reconnaissance d'entités nommées et en intégrant les méthodes et les outils une fois ceux-ci matures (par exemple, l'outil PERDIDO [Gaio et Moncla 2017]). L'idée sera également d'optimiser ces méthodes et de les adapter aux particularités de nos corpus en les fondant sur les analyses critiques des sources et la définition manuelle préliminaire dans celles-ci d'entités à reconnaître et à extraire (comme nous l'avons fait, par exemple, sur des extraits du roman Texaco) (Soudani et al. 2018). Inversement, nous pourrons améliorer la reconnaissance automatique par une reprise manuelle, partant du principe démontré plusieurs fois qu'une première automatisation, même imparfaite, réduit considérablement le temps de reprise manuelle de la numérisation (Soudani et al. 2018).

La deuxième piste pour l'accumulation d'informations géohistoriques sur la ville de Fort-de-France est le recours à des informations numérisées par d'autres, des institutions (bibliothèques, archives, CollEx ${ }^{12}$ ), des chercheurs, des bases et des entrepôts de données ouverts (OSM, Gallica, Data.bnf.fr). On pourrait aussi envisager de faire appel à la numérisation contributive (crowdsourcing) sur le modèle de nombreux projets de numérisation documentaire ${ }^{13}$. Et ce d'autant plus que la cartographie participative offre un intérêt majeur dans une perspective critique, qui plus est postcoloniale (Mericskay et Roche 2011 ; Hirt et Roche 2013).

Une fois ces différentes pistes explorées pour définir et collecter une information géohistorique critique, il faudrait s'attaquer ensuite à la question de comment analyser ces systèmes d'information géohistorique renouvelés. L'exploration visuelle de cartes de traitement que nous avons menée offre un premier outil d'analyse spatiale, et la modélisation atomisée et multidimensionnelle ouvre une multitude de possibilités. Néanmoins, leur seule application ne suffit pas à épuiser l'ensemble des enseignements promis par une modélisation géohistorique. Mais cette question dépasse le cadre de cet article. 


\section{Conclusion}

L'objectif de cet article était de poser les questions soulevées par l'information géohistorique postcoloniale numérique. Elles sont nombreuses et profondes et permettent d'éclairer d'une nouvelle lumière certains problèmes méthodologiques auxquels se confrontent en général la géomatique historique et la modélisation urbaine. Elle engage en outre à adopter une posture résolument interdisciplinaire, à la rencontre de la géographie, de l'histoire, de l'étude critique des représentations, de l'analyse des arts, des discours et des récits ; et ce, tant d'un point de vue théorique, que d'un point de vue méthodologique et technique. Cette ambition ne peut se réaliser que par le dialogue de la géomatique avec la modélisation historique et archéologique, la textométrie et le traitement automatique des langues, ou encore les humanités numériques documentaires. En un mot, il s'agit de s'inscrire résolument dans l'ouverture d'une grande discipline de recherche méthodologique transversale, que l'on peut désigner par l'expression humanités numériques spatialisées.

\section{Bibliographie}

Arnaud, Jean-Luc. 2008. Analyse spatiale, cartographie et histoire urbaine. Marseille : Éditions Parenthèses.

Bernabé, Jean, Patrick Chamoiseau et Raphaël Confiant. 1989. Éloge de la créolité. Paris : Gallimard.

Blais, Hélène. 2014. Mirages de la carte. L'invention de l'Algérie coloniale. Paris : Fayard.

Brando, Carmen, Francesca Frontini et Mathieu Roche, éd. 2018. Actes de l'atelier Humanités numériques spatialisées (HumaNS), conférence SAGEO'2018, Montpellier, 6 novembre. http s://agritrop.cirad.fr/589755/.

Chamoiseau, Patrick. 1986. Chronique des sept misères. Paris : Gallimard.

Chamoiseau, Patrick. 1992. Texaco. Paris : Gallimard.

Devillers, Rodolphe et Robert Jeansoulin. 2005. Qualité de l'information géographique. Paris : Hermès Science Publications et Lavoisier.

Gaio, Mauro et Ludovic Moncla. 2017. « Extended Named Entity Recognition Using FiniteState Transducers : An Application To Place Names ». Dans Proceedings of The Ninth International Conference on Advanced Geographic Information Systems, Applications, and Services (GEOProcessing 2017). Nice, 19-23 mars, édité par Claus-Peter Rückemann, Yerach Doytsher, Jianhong Cecilia Xia et Fernando José Braz. IARA. https://hal.archives-ouvertes.fr/hal-01492994.

Glissant, Édouard. 1981. Le Discours antillais. Paris : Éditions du Seuil.

Harley, Brian. 2001. The New Nature of Maps. Essay on the History of Cartography. Baltimore : The John Hopkins University Press.

Hirt, Irène et Stéphane Roche. 2013. "Cartographie participative ". Dans Dictionnaire critique et interdisciplinaire de la participation, édité par Ilaria Casillo, Rémi Barbier, Loïc Blondiaux, Francis Chateauraynaud, Jean-Michel Fourniau, Rémi Lefebvre, Catherine Neveu et Denis Salles. Paris : GIS Démocratie et participation. https://www.dicopart.$\mathrm{fr} / \mathrm{fr} /$ dico/cartographie-participative.

INSEE. 2016. « Définition des îlots regroupés pour l'information statistique (IRIS) ». insee.fr. https://www.insee.fr/fr/metadonnees/definition/c1523.

Letchimy, Serge. 1992. De l'habitat précaire à la ville : l'exemple martiniquais. Paris : L'Harmattan. 
Mathian, Hélène et Léna Sanders. 2014. Objets géographiques et processus de changement. Approches spatio-temporelles. Paris : ISTE Éditions.

Mericskay, Boris et Stéphane Roche. 2011. « Cartographie 2.0 : le grand public, producteur de contenus et de savoirs géographiques avec le web 2.0 \%. Cybergeo : European Journal of Geography, octobre. https://doi.org/10.4000/cybergeo.24710.

Moncla, Ludovic, Mauro Gaio et Thierry Joliveau. 2018. « Cartographier les odonymes de Paris cités dans les romans du $\mathrm{XIX}^{\mathrm{e}}$ siècle ". Dans Actes de l'atelier Humanités numériques spatialisées (HumaNS), conférence SAGEO'2018, Montpellier, 6 novembre, édité par Carmen Brando, Francesca Frontini et Mathieu Roche, 20-25. https://agritrop.cirad.fr/589755/.

Morel, Juliette, Rémi Crouzevialle et Anne Massoni. 2020. "Construction and Management of a Geo-Historical Information System for an Interdisciplinary and Contributory Atlas : The Historical Atlas of Limousin ». International Journal of Humanities and Arts Computing 14 (1-2) : 27-45. https://doi.org/10.3366/ijhac.2020.0243.

Noucher, Mathieu. 2017. Les Petites Cartes du Web. Analyse critique des nouvelles fabriques cartographiques. Paris : Éditions Rue d'Ulm.

Peuquet, Donna. 1994. «It's about Time : A Conceptual Framework for the Representation of Temporal Dynamics. Geographic Information Systems ». Annals of the Association of the American Geographers 3 (84) : 441-61. https://doi.org/10.1111/j.1467-8306.1994.tb01869.x.

Rodier, Xavier et Laure Saligny. 2010. « Modélisation des objets historiques selon la fonction, l'espace et le temps pour l'étude des dynamiques urbaines dans la longue durée ». $C y$ bergeo : European Journal of Geography, juin. https://doi.org/10.4000/cybergeo.23175.

Soudani, Aicha, Yosra Meherzi, Asma Bouhafs, Francesca Frontini, Carmen Brando, Yoann Dupont et Frédérique Mélanie-Becquet. 2018. " Adaptation et évaluation de systèmes de reconnaissance et de résolution des entités nommées pour le cas de textes littéraires français du $\mathrm{XIX}^{\mathrm{e}}$ siècle ". Dans Actes de l'atelier Humanités numériques spatialisées (HumaNS), conférence SAGEO'2018, Montpellier, 6 novembre, édité par Carmen Brando, Francesca Frontini et Mathieu Roche, 47-51. https://agritrop.cirad.fr/589755/.

\section{Notes}

1 Voir, par exemple, ALPAGE (AnaLyse diachronique de l'espace urbain PArisien : approche GEomatique), qui présente des cartes anciennes géoréférencées et la vectorisation de résultats d'études historiques et archéologiques sur Paris : https://alpage.humanum.fr ; l'Atlas historique du Limousin, webmapping sur l'histoire de Limoges, qui présente des cartes anciennes de la ville de Limoges géoréférencées : http://vafl-s-applirecherche.unilim.fr/carto-web-AHL/; Venice Time Machine, qui compile la numérisation de l'ensemble des archives de la ville de Venise : https:/www.epfl.ch/research/domains/venice-time-machine/.

2 Depuis 1692, Fort-de-France est la capitale administrative de la Martinique et accueille le palais du gouverneur.

3 Ces comptes sont faits sur les cartes anciennes sur lesquelles sont représentés la ville ou le site de Fort-de-France à une échelle supérieure au 1/50 ooo. Ces cartes sont conservées aux archives départementales de la Martinique, à la BNF (accessibles via Gallica, http s://gallica.bnf.fr) et à la Library of Congress de Washington DC (accessibles via son site Internet, https://www.loc.gov). Aucune carte ancienne représentant Fort-de-France à une échelle supérieure au 1/50 ooo n'est accessible via les plateformes spécialisées en cartographie historiques Navigae (https://www.navigae.fr) et Cartomundi (http://www.cartomundi.fr/site/).

4 En 1946, la départementalisation transforma les colonies de la Guyane, la Guadeloupe, la Martinique et la Réunion en départements français. Cela a eu pour effet de les intégrer au territoire français au même titre que les autres départements.

5 L'étude exhaustive des représentations devenant plus difficile au $\mathrm{xx}^{\mathrm{e}}$ siècle, du fait de la multiplication des cartes de la Martinique, nous avons choisi de nous concentrer sur les cartes conservées aux archives départementales de la Martinique sur cette période.

6 Par exemple, la base OpenStreetMap indiquait en avril 2020 moins de 100 établissements scolaires tous niveaux confondus en Martinique (clef OSM = " amenity », valeur = "school »), alors qu'il en existe en réalité 340 dans l'île (académie de Martinique, 20172018). 
7 Cartothèque de l'IGN à Saint-Mandé, archives nationales d'outre-mer (ANOM) à Aix-enProvence, Bibliothèque nationale de France à Paris, etc. Seules 16 séries de cartes représentant Fort-de-France à une échelle supérieure au 1/50 ooo sont conservées sur place, aux archives départementales de la Martinique.

830 cartes de Fort-de-France sont visualisables via Gallica : https://gallica.bnf.fr/services/engine/search/sru?operation=searchRetrieve\&query=(gallica\%20all\%20\%22Fort-de -France\%20Martinique\%22)\%20and\%20dc.type\%20all\%20\%22carte\%22; 13 via le site de la Library of Congress : https://www.loc.gov/maps/?q=Fort-de-France+Martinique ; pas de numérisation des cartes conservées aux ANOM.

9 Texaco a été lauréat du prix Goncourt en 1992.

10 « Foyalais » est le gentilé désignant les habitants de Fort-de-France.

$11 \mathrm{https} / / /$ adresse.data.gouv.fr.

12 Parmi les banques de numérisation documentaire comportant des documents sur les Antilles, on peut citer : la bibliothèque du Réseau francophone numérique qui a pour mission de rassembler et de présenter des fonds documentaires issus du monde francophone, Caraïbes comprises ; le réseau MANIOC (" Manioc : bibliothèque numérique Caraïbe, Amazonie, plateau des Guyanes ", http://www.manioc.org), émanant de la collection d'excellence (CollEx) du SCD de l'université des Antilles ; la banque numérique des patrimoines martiniquais, développée par les archives départementales de Martinique, qui procèdent à la numérisation des archives départementales et à leur mise en ligne ; ou encore la bibliothèque Schœlcher qui diffuse une grande partie de ses collections numérisées par le portail des médiathèques de la Martinique (http://mediatheques.collectivitedemartinique.mq). On peut aussi citer le projet Library of Glissant Studies de la Louisiana State University et de l'université des Antilles qui a pour objectif de créer la bibliographie en ligne la plus exhaustive possible sur Édouard Glissant (https://www.glissantstudies.com).

13 Sur les exemples de l'initiative "Adoptez un livre !" de la Bibliothèque nationale de France pour le financement du développement de la bibliothèque numérique Gallica; de l'application Building Inspector de la New York Public Library qui invite n'importe qui à devenir " citizen cartographer " pour améliorer et valider la vectorisation automatique qui a été faite des bâtiments présents sur les cartes anciennes de New York ; de l'initiative " Adopter un texte» du Labex Obvil de l'UMR CELLF (Sorbonne-Université), qui invitait les doctorants en littérature à en baliser la structure en XML et à l'encoder selon les normes de la Text Encoding Initiative (TEI). On peut encore citer l'incitation à la contribution pour le référencement bibliographique des œuvres de et sur Édouard Glissant dans le cadre du projet Glissant Studies ou l'outil collaboratif de transcription numérique des archives historiques écrites et des matériaux de terrain des sciences humaines et sociales offert par la plateforme Transcrire d'Huma-Num.

\section{Auteur}

\section{Juliette Morel}

UR 3482 Lab'Urba, université Paris-Est-Créteil, Créteil, France

Après une thèse de littérature consacrée à la modélisation de la spatialité du récit dans l'œuvre de l'auteur algérien Kateb Yacine (2016), Juliette Morel a travaillé avec des historiens pour développer un système d'information géographique et historique et une interface de visualisation cartographique sur l'histoire de la ville de Limoges (université de Limoges, 2017-2018). Depuis 2020, elle est maîtresse de conférence en géographie à l'université Paris-Est-Créteil, spécialiste du dialogue entre géomatique et humanités numériques.

ORCID : 0000-0002-2028-0658

juliette.morel@u-pec.fr

\section{Droits d'auteur}

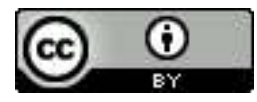

Les contenus de la revue Humanités numériques sont mis à disposition selon les termes de la Licence Creative Commons Attribution 4.0 International. 